ISSN 1112-9867

Available online at

http://www.jfas.info

\title{
DURABILITY OF NATURAL POZZOLAN-BASED MORTAR EXPOSED TO SULFATE ATTACK
}

\author{
L. Laoufi ${ }^{1, *}$, Y. Senhadji ${ }^{1}$, A. Benazzouk ${ }^{2}$, T. Langlet $^{2}$, M. Mouli ${ }^{3}$ and I. Laoufi ${ }^{1}$ \\ ${ }^{1}$ Département de Génie Civil, Université de Mascara, Mascara 29000, Algérie \\ ${ }^{2}$ Laboratoire des Technologies Innovantes, Université de Picardie, Amiens, France \\ ${ }^{3}$ Département de Génie Civil, Laboratoire Lab-Mat, ENP-Oran El Mnaouer 31000, Algérie
}

Received: 14 December 2015 / Accepted: 05 Febraury 2016 / Published online: 01 May 2016

\begin{abstract}
Cement is a strategic commodity in the civil engineering for the construction of reinforced concrete structures. But its production generates around $5 \%$ of toxic gases such as $\mathrm{CO}_{2}$ responsible for environmental degradation. Furthermore, cement industry is a consumer sector of non-renewable energy. The use in the cement of natural additions is a solution to reduce the $\mathrm{CO}_{2}$ gas and the cost of production. The purpose of this work is the study of a sustainable building material: natural pozzolan Beni-saf (PNB) incorporated to mortars exposed to sulfate attack $\left(5 \% \mathrm{Na}_{2} \mathrm{SO}_{4}\right)$. The loss of mass, monitoring the $\mathrm{pH}$ reading of each attack solution as well as specimens dimensions are different tests to study the durability of mortars made with 10, 20 and $30 \%$ of natural pozzolan. The result derived from this research is that pozzolan improves mortars resistance to sodium sulfate environment.
\end{abstract}

Keywords: natural pozzolan; chemical attack; durability; sodium sulfate; $\mathrm{pH}$; mass loss.

Author Correspondence, e-mail: laoufifr@yahoo.fr

doi: http://dx.doi.org/10.4314/jfas.v8i2.7 


\section{INTRODUCTION}

La durabilité d'un ouvrage dépend de nombreux paramètres dont la qualité de sa conception, la qualité des matériaux et des produits utilisés, la qualité des dispositions constructives, de la réalisation de l'ouvrage et de la mise en œuvre des produits ainsi que des diverses conditions d'usage, d'exploitation et de maintenance. Il est possible désormais de définir des objectifs de durabilité et de choisir avec précision les caractéristiques du béton en fonction de l'agressivité du milieu dans lequel se trouve l'ouvrage et d'optimiser ses caractéristiques afin de les adapter à la durée d'utilisation souhaitée.

L'eau de l'extérieur peut pénétrer dans le réseau de pores et de capillaires du béton et apporter des ions agressifs qui peuvent réagir avec les hydrates et changer leur structure. Les

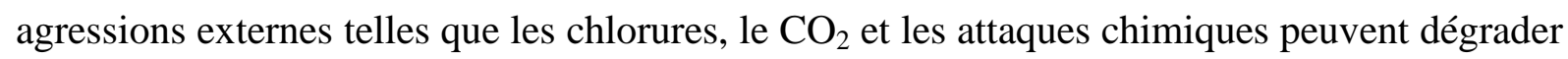
les propriétés physiques et mécaniques du béton ainsi que sa durabilité au cours du temps. Les attaques chimiques telles que les attaques sulfates dégradent les mortiers et bétons; ces sulfates peuvent d'ailleurs provenir aussi bien des zones industrielles que de l'activité urbaine. Cependant, ce n'est que lorsque ces sulfates sont dissous dans l'eau (souterraine ou autre) qu'ils présentent un danger potentiel pour pénétrer dans la matrice du ciment, réagir avec les hydrates et changer leur structure [1].

Les pouzzolanes naturelles influent positivement sur la durabilité des mortiers vis-à-vis de l'attaque des sulfates. Il est généralement admis que les pouzzolanes ont besoin de moins d'énergie pour leur broyage que le clinker et que l'utilisation de matériaux pouzzolaniques dans le ciment ou dans le béton entraîne de nombreuses propriétés bénéfiques, comme la faible chaleur d'hydratation, l'élévation de la résistance à la compression, la faible perméabilité, la haute résistance aux sulfates et la faible activité de l'alcali-silice. L'utilisation du ciment Portland avec de la pouzzolane naturelle génère une réaction entre l'hydroxyde de calcium, produit par l'hydratation du ciment Portland et le $\mathrm{SiO}_{2}$ amorphe de la phase vitreuse de la pouzzolane. Ceci conduit à une augmentation de la formation du gel de l'hydrate de silicate de calcium [C-S-H] dit de 2éme génération et ainsi à l'augmentation de la résistance et de la densité [2]. Dans cette étude, l'effet de $5 \% \mathrm{Na}_{2} \mathrm{SO}_{4}$ sur la perte de masse, le suivi du pH et des dimensions d'éprouvettes en mortiers pouzzolaniques et en mortiers témoin ont été 
examinés.

\section{ETUDE EXPERIMENTALE}

\subsection{Les Matériaux}

\subsubsection{Le Ciment}

Le ciment utilisé dans tous les essais est un ciment Portland ordinaire type CEM I - 42.5 N fabriqué selon la norme Algérienne NA 442-2008 [3], provenant de la cimenterie de Zahana (Algérie). Les propriétés physiques et la composition minéralogique du ciment sont représentées par le Tableau 1. La composition chimique du ciment est représentée par le Tableau 2.

Tableau 1. Propriétés physiques et composition minéralogique du ciment

\begin{tabular}{|c|c|c|c|c|c|c|}
\hline \multicolumn{3}{|c|}{ Propriétés physiques } & \multicolumn{4}{|c|}{ Composition minéralogique } \\
\hline Surface spécifique & masse volumique & densité & $\mathrm{C}_{2} \mathrm{~S}$ & $\mathbf{C}_{3} \mathbf{S}$ & $\mathbf{C}_{3} \mathrm{~A}$ & $\mathrm{C}_{4} \mathrm{AF}$ \\
\hline Blaine $\left(\mathrm{cm}^{2} / \mathrm{g}\right)$ & apparente $\left(\mathrm{g} / \mathrm{cm}^{3}\right)$ & & \multicolumn{4}{|c|}{$(\%)$} \\
\hline 3308 & 1.02 & 3.12 & 25.69 & 47.15 & 2.41 & 15.12 \\
\hline
\end{tabular}

Tableau 2. Composition chimique élémentaire du ciment (\%)

\begin{tabular}{llllllllll}
\hline $\mathbf{C a O}$ & $\mathrm{SiO}_{2}$ & $\mathrm{Al}_{2} \mathrm{O}_{3}$ & $\mathrm{Fe}_{2} \mathrm{O}_{3}$ & $\mathrm{SO}_{3}$ & $\mathrm{~K}_{2} \mathrm{O}$ & $\mathbf{N a}_{2} \mathrm{O}$ & $\mathrm{MgO}$ & $\mathrm{RI}$ & PAF \\
\hline 63,89 & 21,4 & 4,59 & 5,52 & 2,72 & 0,41 & 0,13 & 1,37 & 0,22 & 2,47 \\
\hline
\end{tabular}

RI : Résidu insoluble, PAF : Perte au feu.

\subsubsection{La Pouzzolane Naturelle}

Après un séchage de $105^{\circ} \mathrm{C}$ pour éliminer l'eau libre, la pouzzolane a été broyée finement. Les propriétés physiques et la compostion chimique de la pouzzolane naturelle de béni-Saf (PNB) sont représentées par les Tableaux 3 et 4 respectivement.

Tableau 3. Propriétés physiques de la PNB

\begin{tabular}{ccc}
\hline $\begin{array}{c}\text { Surface spécifique } \\
\text { Blaine }\left(\mathbf{c m}^{2} / \mathbf{g}\right)\end{array}$ & $\begin{array}{c}\text { masse volumique } \\
\text { apparente }\left(\mathbf{g} / \mathbf{c m}^{\mathbf{3}}\right)\end{array}$ & densité \\
\hline 4255 & 0.98 & 2.75 \\
\hline
\end{tabular}


Tableau 4. Composition chimique de la PNB (\%)

\begin{tabular}{cccc}
\hline $\mathbf{S i O}_{2}$ & $\mathbf{A l}_{2} \mathbf{O}_{3}$ & $\mathbf{F e}_{2} \mathbf{O}_{\mathbf{3}}$ & $\mathbf{C a O}$ \\
\hline 45.90 & 17.10 & 10.60 & 10.20 \\
\hline
\end{tabular}

\section{PREPARATION DES ECHANTILLONS ET PROCEDURE DES ESSAIS}

Les mortiers destinés à la confection des éprouvettes de dimensions $(5 \times 5 \times 5) \mathrm{cm}^{3}$ furent mélangés et préparés avec un ciment Portland ordinaire type CEM I-42.5 N selon les procédures de la norme NF EN 196-1 [4]. Les taux ciment/sable et eau/ciment furent pris égaux respectivement à 1:3 et 1:2 pour toutes les éprouvettes.

Les éprouvettes en nombre de douze par solution soit trois éprouvettes pour chaque mélange de mortier, seront démoulées après 24 heures, et conservées dans l'eau saturée en chaux pendant 28 jours à une température de $20 \pm 2^{\circ} \mathrm{C}$, avant d'être soumises à l'attaque sulfatique. Après cette période de cure, les éprouvettes seront pesées pour déterminer leur poids initial avant l'attaque chimique, puis immergées dans les solutions à $5 \%$ de $\mathrm{Na}_{2} \mathrm{SO}_{4}$. L'évaluation de la mesure de la perte de masse des échantillons se fera comme suit :

Perte de masse $\left.(\%)=\left[\left(\mathrm{M}_{1}-\mathrm{M}_{2}\right) / \mathrm{M}_{1}\right)\right] \times 100$

Où $\mathrm{M}_{1}$ est le poids de l'éprouvette avant immersion et $\mathrm{M}_{2}$ le poids de l'éprouvette nettoyée et séchée après immersion. Les éprouvettes préparées avec le ciment type CEM I- $42.5 \mathrm{~N}$ et 0, 10, 20 et $30 \%$ de PNB en masse de ciment furent dénommés M0, M10, M20 et M30 respectivement.

\subsection{Maniabilité des Mortiers Frais}

Les maniabilités des mortiers frais furent déterminées par la table à secousse selon la norme ASTM C 1437 [5] avec une quantité d'eau constante pour la préparation de toutes les mélanges de pâtes $(E / L=0.5)$ et les valeurs des résultats sont données dans le Tableau 5. La maniabilité des mortiers pouzzolaniques diminue avec l'augmentation du taux de substitution du ciment par la pouzzolane naturelle. 
Tableau 5. Influence de la PNB sur la maniabilité des mortiers

\begin{tabular}{cccc}
\hline $\begin{array}{c}\text { Désignation } \\
\text { de }\end{array}$ & $\begin{array}{c}\text { Pouzzolane } \\
\text { maturelle }(\%)\end{array}$ & E/L & $\begin{array}{c}\text { Etalement } \\
(\mathbf{m m})\end{array}$ \\
\hline $\mathrm{M}_{0}$ & 0 & 0,5 & 115 \\
\hline $\mathrm{M}_{10}$ & 10 & 0,5 & 103 \\
\hline $\mathrm{M}_{20}$ & 20 & 0,5 & 100 \\
\hline $\mathrm{M}_{30}$ & 30 & 0,5 & 93 \\
\hline
\end{tabular}

\subsection{Consistance et Temps de Prise}

Les résultats de ces essais physiques de caractérisation des pâtes normalisées de ciment avec et sans PNB sont présentés dans le Tableau 6. On voit clairement dans ce tableau que les pâtes de ciment avec différents taux de pouzzolane ont besoin plus d'eau pour l'obtention d'une consistance analogue à celle de la pate sans PNB (P0), en outre, plus le pourcentage de PNB est grand et plus la demande en eau est grande. Cette augmentation de la consistance normale des pates pouzzolaniques est due probablement à l'augmentation progressive de la surface spécifique du liant ce qui entraine naturellement un fort appel aux molécules d'eau afin de mouiller toute la pate.

Tableau 6. Influence de la PNB sur la consistance normale et le temps de prise

\begin{tabular}{lcccc}
\hline $\begin{array}{c}\text { Désignation des } \\
\text { pâtes }\end{array}$ & $\begin{array}{c}\text { Pouzzolane } \\
\text { naturelle }(\boldsymbol{\%})\end{array}$ & $\begin{array}{c}\text { Consistance } \\
(\mathbf{m m})\end{array}$ & $\begin{array}{c}\text { Début de prise } \\
(\mathbf{h}: \mathbf{m i n})\end{array}$ & $\begin{array}{c}\text { fin de prise } \\
\text { (h : } \mathbf{m i n})\end{array}$ \\
\hline P0 & 0 & 24.0 & $2: 23$ & $3: 45$ \\
\hline P10 & 10 & 25.0 & $2: 35$ & $3: 57$ \\
\hline P20 & 20 & 25.5 & $2: 50$ & $4: 10$ \\
\hline P30 & 30 & 26.0 & $3: 25$ & $4: 15$ \\
\hline
\end{tabular}

$\mathrm{PX}=$ Pate à $\mathrm{X} \%$ de PNB.

\section{RESULTATS ET ANALYSE}

\subsection{Action du Sulfate de Sodium}

La Figure 1 montre la variation de la masse des éprouvettes de mortier M0, M10, M20 et M30 confectionnées avec et sans ajout de la PNB avec différents pourcentages 0, 10, 20 et 30\% respectivement, en fonction de la période d'immersion dans la solution de $5 \% \mathrm{Na}_{2} \mathrm{SO}_{4}$. 


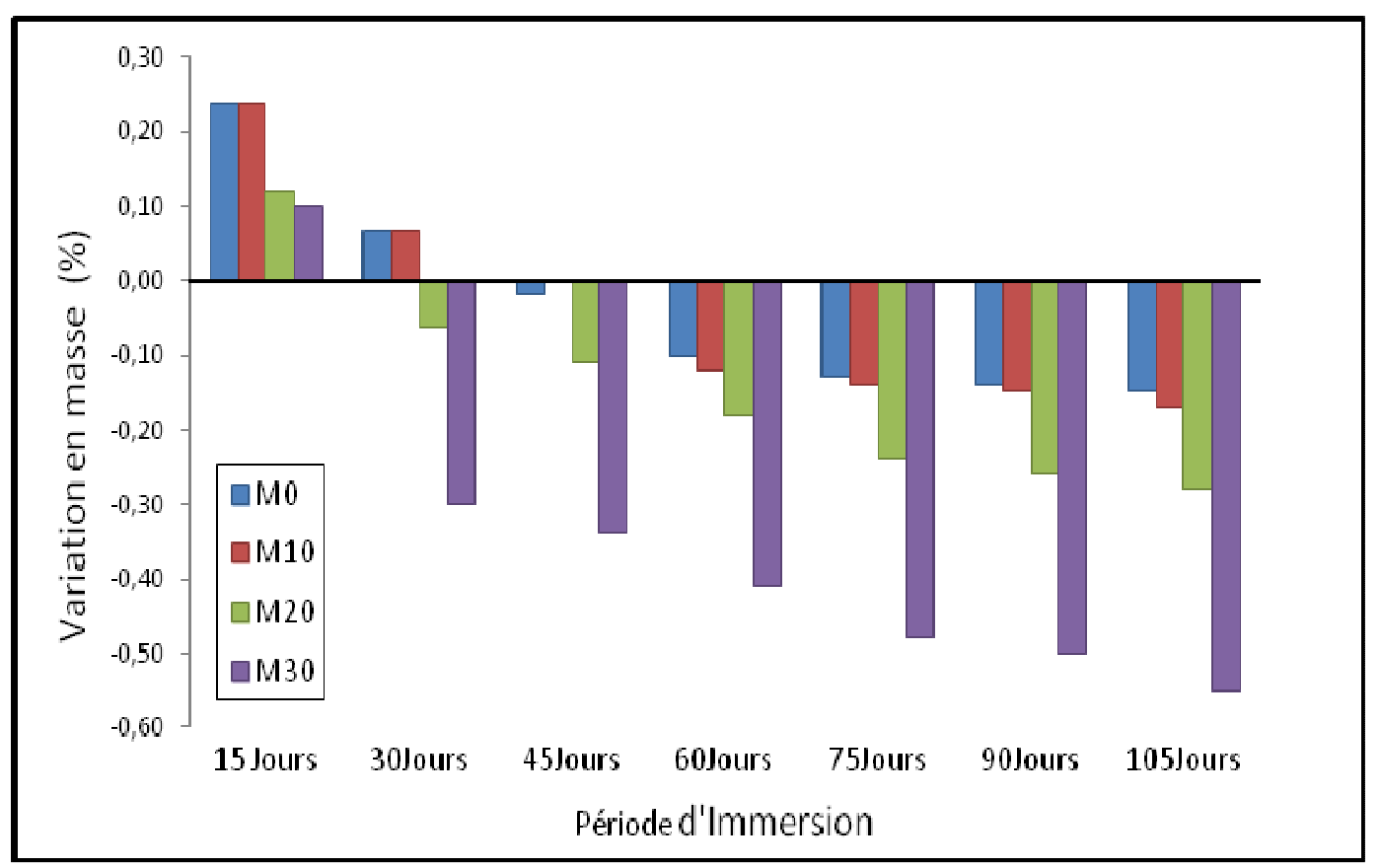

Fig.1. Variation en masse des différents mortiers en fonction de la période d'immersion dans une solution de $5 \% \mathrm{Na}_{2} \mathrm{SO}_{4}$

Dans la Figure 1, on constate, d'une façon générale, que l'ensemble des mortiers ont marqué des gains de masse permanents à partir de la 4ème semaine de conservation qui sont représentés par des valeurs de signe négatif. Avant cette date, des faibles lectures de perte de masse ont été identifiées pour les quatre types de mortiers, et sont représentées par des signes positifs.

A 15 jours, période où la réaction pouzzolanique est inactive, on enregistre des réductions des pertes de masse pour les mortiers pouzzolaniques M20 et M30 qui sont de 0.12 et $0.10 \%$ respectivement, comparativement au mortier M0 sans pouzzolane, ce dernier enregistre une perte de masse de $0.24 \%$. Torri et al [6], confirment que la dégradation progressive et la dissolution de la matrice du ciment au jeune âge sont attribuées à la décomposition de l'hydroxyde de calcium et par conséquent à la formation d'une quantité de gypse qui est dissoute, selon la réaction suivante:

$$
\mathrm{Ca}(\mathrm{OH})_{2}+\mathrm{Na}_{2} \mathrm{SO}_{4}+\mathrm{H}_{2} \mathrm{O} \rightarrow \mathrm{CaSO}_{4} \cdot 2 \mathrm{H}_{2} \mathrm{O}+2 \mathrm{NaOH}
$$

Portlandite gypse

Par contre, à l'âge de 105 jours, les augmentations de la masse des mortiers pouzzolaniques M10, M20 et M30 sont appréciables et sont $0.17,0.28$ et $0.55 \%$ respectivement par rapport au mortier sans pouzzolane M0, ce dernier enregistre un gain de masse de $0.15 \%$ seulement. 
Cette augmentation de la masse des mortiers immergés en solutions sulfatiques peut être expliquée par la formation de produits avec une faible solubilité et qui sont bloqués à l'intérieur de la matrice, ce qui explique le gain de poids. Les produits qui résultent de ces réactions sont l'ettringite et le gypse. Notons que la réaction pouzzolanique à cette période de 105 jours commence à réagir, la PNB fixe la chaux libérée lors de l'hydratation du ciment, pour former un gel de C-S-H qui colmate et densifie la matrice cimentaire. Donc l'incorporation de la PNB a été bénéfique pour le mortier contre l'attaque du sulfate de sodium $\left(5 \% \mathrm{Na}_{2} \mathrm{SO}_{4}\right)$. Ce résultat confirme celui de Senhadji [7] qui a travaillé sur le comportement des mortiers à base de la PNB vis-à-vis des attaques sulfates.

\subsection{Le Suivi de la Lecture du pH de la Solution d'Attaque $5 \% \mathrm{Na}_{2} \mathrm{SO}_{4}$}

Le mortier est un solide poreux, qui renferme dans ses pores une solution interstitielle enrichie en alcalis dont le pH varie de 12,5 à 13,5. Le système chimique du mortier, avec son pH élevé, est presque toujours en déséquilibre avec son environnement, dont le $\mathrm{pH}$ est bien inférieur. L'eau de l'extérieur pénètre à travers le système des pores et des capillaires, même dans un mortier non fissuré, entraînant une diminution de son $\mathrm{pH}$. Chaque enrichissement en sulfates à l'intérieur de la pâte hydratée cause aisément la transformation du monosulfoaluminate hydraté ou des aluminates hydratés, qui sont des produits finaux de l'hydratation du ciment, en ettringite, ou dans le cas d'une concentration en sulfates élevée ou d'un manque d'aluminates, la portlandite se transformera en gypse [8].
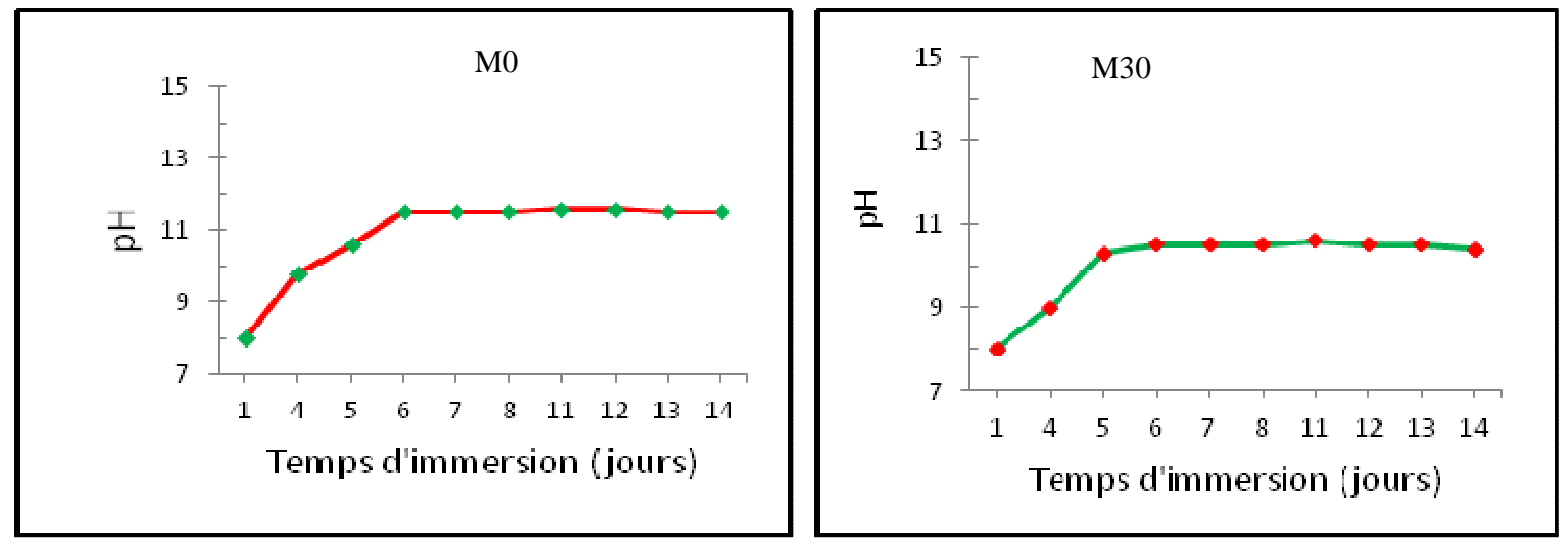

Fig.2. Variation du $\mathrm{pH}$ au cours du temps des mortiers $\mathrm{M} 0$ et $\mathrm{M} 30$ dans $5 \% \mathrm{Na}_{2} \mathrm{SO}_{4}$ 
La Figure 2 présente les relevés de ces $\mathrm{pH}$ au cours des premiers 14 jours pour les mortiers M0 et M30. L'ensemble des mortiers présentent une allure de $\mathrm{pH}$ croissante jusqu'au sixième jour et une allure uniforme au-delà de cette date. Durant cette période, le $\mathrm{pH}$ relevé au $1^{\mathrm{er}}$ jour à une valeur de 8 pour les deux mortiers, il atteint 11.5 et 10.4 pour M0 et M30 au quatorzième jour, respectivement. Nous constatons donc que le $\mathrm{pH}$ du mortier sans pouzzolane $\mathrm{M} 0$, durant cette période, a augmenté plus rapidement que le mortier pouzzolanique M30, cela veut dire que c'est la pouzzolane naturelle qui a joué un rôle important dans les réactions de transformation puisque c'est le seul facteur qui change entre M0 et M30.

L'augmentation des valeurs du $\mathrm{pH}$ représente une perte de concentration de la solution sulfatique suite à sa réaction chimique avec les composants de la matrice cimentaire du mortier pour former le gypse par dissolution de la chaux hydratée $\mathrm{Ca}(\mathrm{OH})_{2}$ et d'hydroxyde de sodium $(\mathrm{NaOH})$ comme indiqué dans l'équation (Eq. 2). L'hydroxyde de sodium étant basique, il est normal que le pH augmente. Ceci est en accord avec les résultats de recherches de Senhadji [7], Yeginobali et Dilek [9] et Laoufi [10].

\subsection{Suivi des Volumes des Eprouvettes}

Le suivi et la mesure des dimensions des éprouvettes immergées dans la solution sulfate 5\% $\mathrm{Na}_{2} \mathrm{SO}_{4}$ est effectué manuellement par un pied à coulisse. Le calcul des volumes des éprouvettes aux échéances fixées est un moyen pour évaluer les dégradations de l'aspect extérieur des éprouvettes et de confirmer et comparer les résultats obtenus, Figure 3.

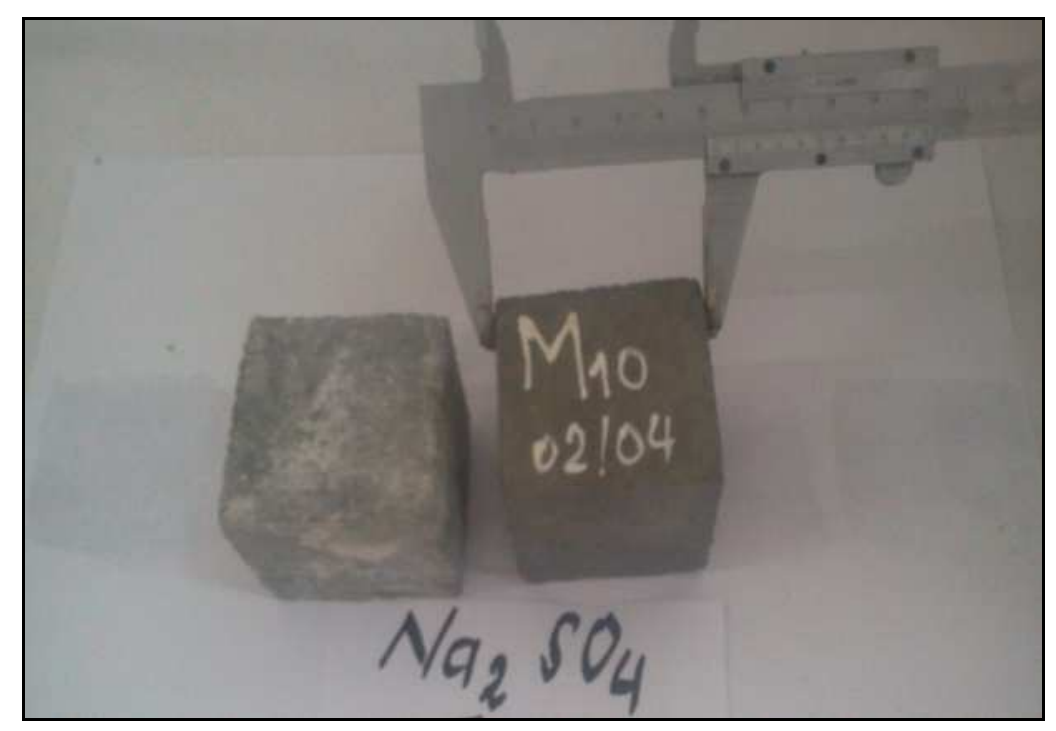

Fig.3. Mesure des dimensions d'une éprouvette à l'aide d'un pied à coulisse 
La Figure 4, représente le suivi des volumes, au cours du temps, des éprouvettes de mortier utilisées dans cet essai. Il y'a lieu de constater une faible variation des dimensions des éprouvettes représentant les volumes. A l'âge de 105 jours les volumes des mortiers M0, M10, M20 et M30 ont diminué d'environ 0.1, 0.1, 0.3 et $0.1 \%$ respectivement. Cela veut dire que la solution $5 \% \mathrm{Na}_{2} \mathrm{SO}_{4}$ n'a pas influé sur les éprouvettes pendant toute la période d'exposition.

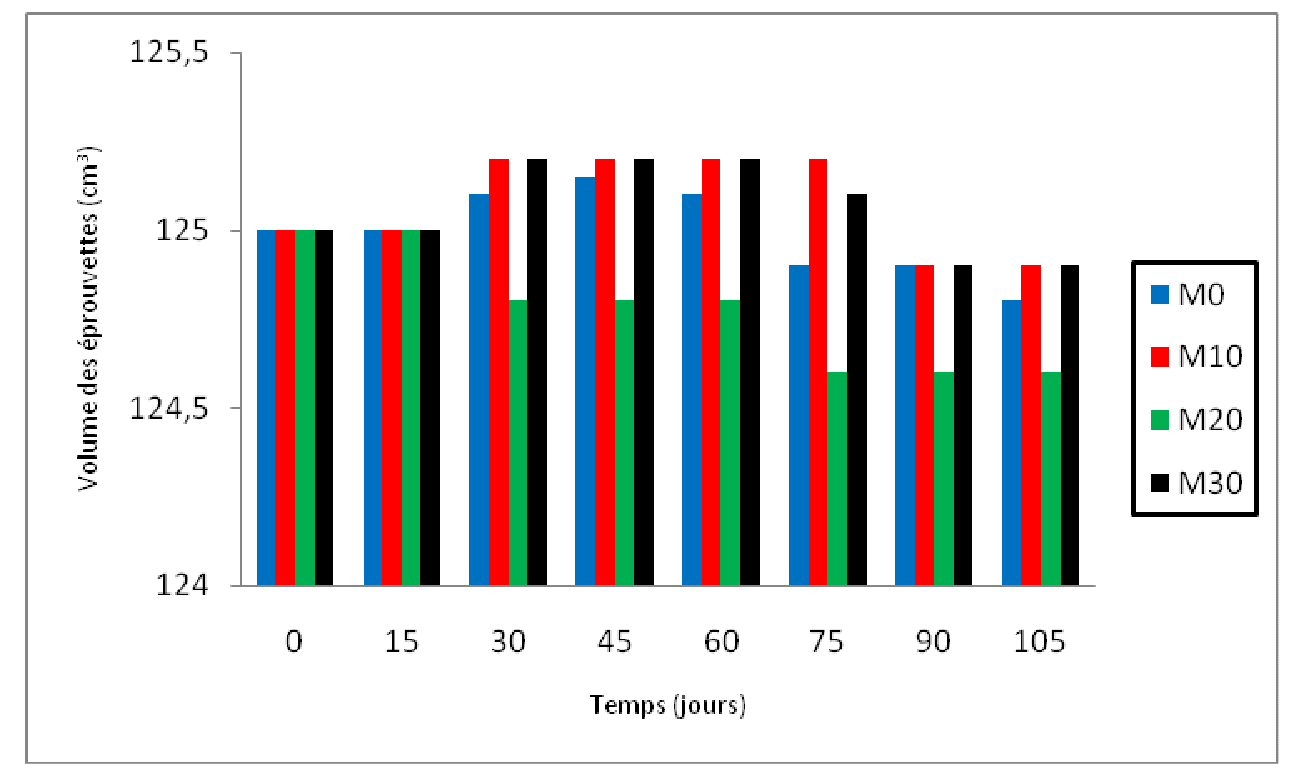

Fig.4. Suivi des volumes des éprouvettes de mortiers exposés à l'attaque $5 \% \mathrm{Na}_{2} \mathrm{SO}_{4}$ au cours du temps

Les résultats de faibles pertes en volume et le gain de masse de l'ensemble des mortiers qui en résulte, peuvent être expliqués par le blocage à l'intérieur de la matrice cimentaire des produits formés suite au mécanisme d'attaque des mortiers par les sulfates; il y a eu un colmatage des pores existant dans le mortier empêchant ainsi l'éjection des produits formés vers l'extérieur.

\subsection{Examen Visuel}

L'examen visuel des mortiers ayant subits des attaques chimiques en contact avec l'acide sulfatique est un moyen pour évaluer la détérioration des propriétés physiques et les dégradations de l'aspect extérieur des éprouvettes, et de confirmer et comparer les résultats obtenus. Les éprouvettes immergées à l'âge de 105 jours dans la solution sulfatique de sodium $5 \% \mathrm{Na}_{2} \mathrm{SO}_{4}$, ne révèlent aucun signe de dégradation, Figure 5. 


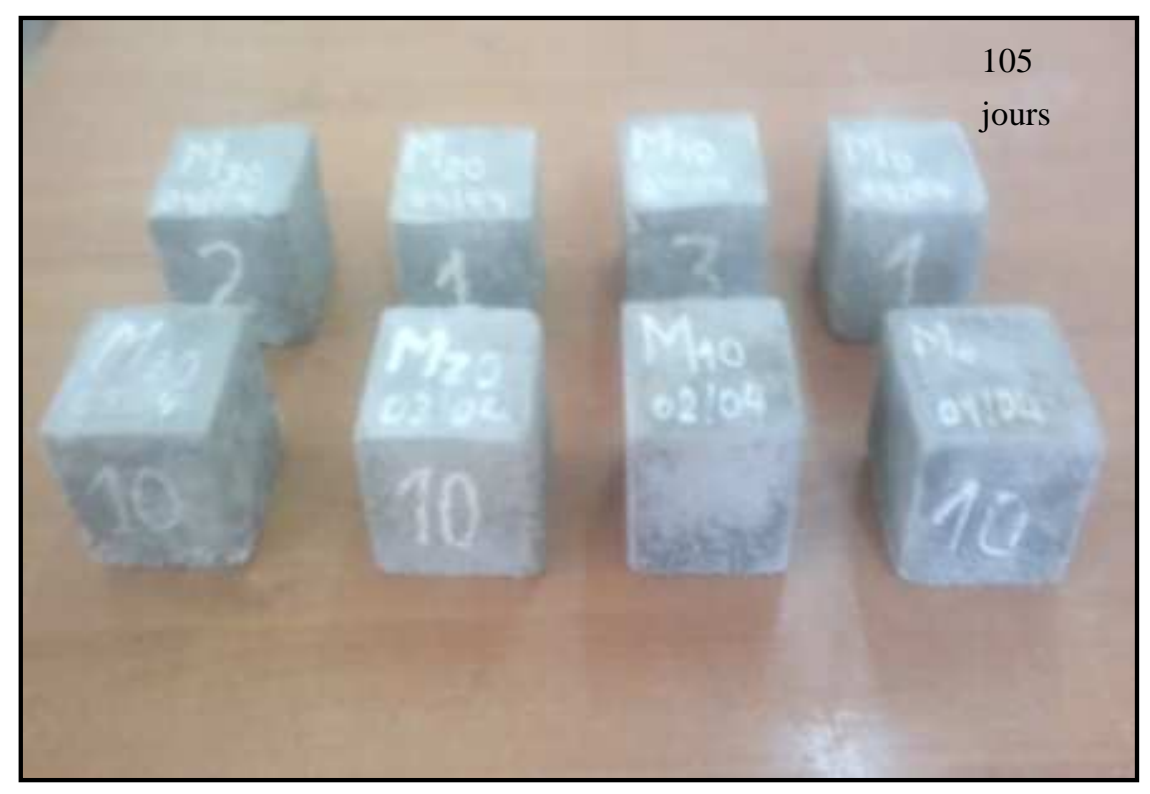

Fig.5. Etat des éprouvettes M0, M10, M20 et M30 (de droite à gauche) dans $5 \% \mathrm{Na}_{2} \mathrm{SO}_{4}$ comparées à celle exposées dans l'eau (en arrière plan).

\section{CONCLUSION}

A la lumière des essais réalisés au cours de ce travail de recherche nous pouvons tirer les principales conclusions suivantes :

L'incorporation de la pouzzolane naturelle d'Algérie apporte des gains de masse substantiels et est donc bénéfique pour la résistance chimique du ciment pouzzolanique vis-à-vis des ions $\mathrm{SO}_{4}{ }^{2-}$. L'examen visuel des éprouvettes et la faible variation des volumes, au terme des essais, a montré des éprouvettes sans dégradation apparente, preuve que les ions sulfatent présents dans la solution n'ont eu aucun effet négatif sur les mortiers pouzzolaniques.

La maniabilité des pates pouzzolaniques diminue avec l'augmentation du taux de substitution du ciment par la pouzzolane naturelle. En outre les pates pouzzolaniques ont besoin plus d'eau pour l'obtention d'une consistance normale, influent sur le processus d'hydratation du ciment et augmentent les temps de début et de fin de prise proportionnellement aux pourcentages de la pouzzolane. 


\section{REMERCIEMENTS}

Nous remercions le ministère de l'enseignement supérieur et de la recherche scientifique pour les subventions allouées à cette étude de recherche ainsi que celles de la CNEPRU $\mathrm{n}^{\mathrm{o}}$ : J0405520060009. Les auteurs apprécient la collaboration de Mr Siyoucef M. et Mme Boulenoir Z. dans ce travail de recherche. Nous remercions également Mr le professeur T. Langlet et Mr le Dr A. Benazzouk pour nous avoir ouvert les portes de leur laboratoire des matériaux innovants (EA 3899), de l'université d'Amiens (France).

\section{REFERENCES}

[1] Higgins DD, Cem. Concr. Compo., 2003, 25, 913-919. doi:10.1016/S0958-9465(03)00148-3.

[2] Liu B, Xie Y, Li J, Cem. Concr. Res., 2005, 35, 994-998. doi:10.1016/j.cemconres.2004.05.044.

[3] NA 442-08, Liants Hydrauliques-Ciments Courants, Composition, "Spécification et Critères de Conformité", 2008, IANOR, Alger.

[4] NF EN 196-1-06, Méthodes d'essais des ciments-Partie 1: détermination des résistances mécaniques. 2006.

[5] ASTM C 1437-01. Standard Test Method for Flow of Hydraulic Cement Mortar, 2001.

[6] Torri K, Kawamura M, Cement Concrete Research., 1994, 24(2), 361-570. doi:10.1016/0008-8846(94)90063-9

[7] Senhadji Y, L'influence de la nature du ciment sur le comportement des mortiers vis-à-vis des attaques chimiques (acides et sulfates). Thèse de doctorat Université des Sciences et de la Technologie Mohammed Boudiaf (USTMB), Oran, 2013.

[8] Barbarulo R, Comportement des matériaux cimentaires : actions des sulfates et de la température. Thèse de doctorat. Ecole Normale Supérieure de Cachan. Université Laval, 2002. [9] Yeginobali A, Dilek FT, Sulfate Resistance of Mortars Containing Silica Fume as Evaluated by Different Methods, Fly Ash, Silica Fume, Slag, and Naturel Pouzzolans Concrete, Proceeding, Fifth International Conference, Milwaukee, Wisconsin, USA, 1995, 2, 795-813. 
[10] Laoufi L, Comportement Mécanique et Structurel du Béton dans un Environnement Agressive, thèse de doctorat, ENPOran, Algérie (2015).

\section{How to cite this article:}

Laoufi L, Senhadji Y, Benazzouk A, Langlet T, Mouli M and Laoufi I. Durability of Natural Pozzolan-based mortar exposed to sulfate attack. J. Fundam. Appl. Sci., 2016, 8(2), 268-279. 